\title{
Activity of the antiarrhythmic drug amiodarone against Leishmania (L.) infantum: an in vitro and in vivo approach
}

\author{
Erika G. Pinto ${ }^{1 *}$ and Andre G. Tempone ${ }^{2}$
}

\begin{abstract}
Background: Considering the high toxicity and limited therapies available for treating visceral leishmaniasis $(\mathrm{VL})$, the drug repositioning approach represents a faster way to deliver new therapies to the market.

Methods: In this study, we described for the first time the activity of a potent antiarrhythmic, amiodarone (AMD), against $L$. (L.) infantum and its in vitro and in vivo activity.

Results: The evaluation against promastigotes has shown that amiodarone presents leishmanicidal effect against the extracellular form, with an $I_{50}$ value of $10 \mu \mathrm{M}$. The activity was even greater against amastigotes in comparison with promastigotes with an $I_{50}$ value of $0.5 \mu \mathrm{M}$. The selectivity index in relation to the intracellular form demonstrated that the antiparasitic activity was approximately 56 times higher than its toxicity to mammalian cells. Investigation of the in vivo AMD activity in the L. infantum-infected hamster model showed that 51 days after the initial infection, amiodarone was unable to reduce the parasite burden in the spleen and liver when treated for 10 consecutive days, intraperitoneally, at $50 \mathrm{mg} / \mathrm{kg} / \mathrm{day}$, as determined by qPCR. Although not statistically significant, AMD was able to reduce the parasite burden by $20 \%$ in the liver when treated for 10 consecutive days, orally, at $100 \mathrm{mg} / \mathrm{kg} /$ day; no reduction in the spleen was found by qPCR.

Conclusions: Our findings may help further drug design studies seeking new AMD derivatives that may provide new candidates with an in vitro selectivity close to or even greater than that observed in the prototype delivering effectiveness in the experimental model of VL.
\end{abstract}

Keywords: Leishmania, Visceral leishmaniasis, Amiodarone, In vitro, In vivo

\section{Background}

Leishmaniasis is a neglected tropical disease (NTD) caused by the protozoan parasite Leishmania spp., associated with underdeveloped and/or developing countries, presenting infections that manifest especially in tropical or subtropical climates and, consequently, reaching less favored population [1]. Leishmaniasis is called neglected, amongst others factors, because it does not attract the interest of the pharmaceutical sector, which does not consider the market potential sufficient for the investment necessary to develop new drugs [2-4].

\footnotetext{
* Correspondence: e.g.pinto@dundee.ac.uk

${ }^{1}$ Wellcome Centre for Anti-Infectives Research, School of Life Sciences,

University of Dundee, Dundee, UK

Full list of author information is available at the end of the article
}

Leishmaniases affect a total of 98 countries, 3 territories and 5 continents, presenting endemic transmission and totaling more than 58,000 cases of visceral leishmaniasis and 220,000 cases of cutaneous leishmaniasis per year. There is also an overall global estimate of 0.2-0.4 million cases for the visceral form and $0.7-1.2$ for the cutaneous form, making leishmaniases one of the six major endemics for the World Health Organization (WHO) [5, 6].

The chemotherapy for leishmaniases is limited and generally ineffective. The arsenal available is based on a few drugs such as pentavalent antimonial, amphotericin $\mathrm{B}$ and miltefosine, which in most cases result in severe side effects and potential treatment abandonment, enabling the appearance of resistant strains [7]. It is also important to highlight that all the drugs cited above

(c) The Author(s). 2018 Open Access This article is distributed under the terms of the Creative Commons Attribution 4.0 International License (http://creativecommons.org/licenses/by/4.0/), which permits unrestricted use, distribution, and 
were introduced in the leishmaniasis clinic as a result of drug repositioning strategy.

The search for new drugs may require long-term study, large financial resources and a high investment risk. In view of the problematic treatment of visceral leishmaniasis, the screening of drugs developed for another purpose, that is, drugs already available in the clinic to treat other diseases, has been presented as one of the fastest and most effective approaches for introducing new therapies, known as drug repurposing. The repositioning of FDA-approved drugs also stands out as a strategy of lower cost in the medium term with vast examples in the therapy of leishmaniasis $[7,8]$.

The drug chosen in this study was amiodarone (AMD), which was first synthesized as a coronary vasodilator more than 50 years ago and has been widely used as a potent antiarrhythmic [9]. Additionally, AMD activity has been previously reported within in vitro studies using cutaneous forms of leishmaniasis [10-12].

\section{Methods}

\section{Drugs and chemicals}

3-[4,5-Dimethylthiazol-2-yl]-2,5-diphenyl tetrazolium bromide (Thiazol blue; MTT), M-199 medium, miltefosine, RPMI-PR-1640 medium (without phenol red) and sodium dodecyl sulfate (SDS) were purchased from Sigma-Aldrich (St Louis, MO). Amiodarone was kindly donated by Prof. Dr. Humberto Gomes Ferraz (University of Sao Paulo - Brazil).

\section{Bioassay procedures}

BALB/c mice and Golden hamsters (Mesocricetus auratus) were obtained from the animal breeding facility at Adolfo Lutz Institute (São Paulo, Brazil). The animals were maintained in sterilized cages with water and food given ad libitum. Animal procedures were performed with the approval of the Research Ethics Commission (CEUA IAL 04/2011) and in agreement with the Guidelines for the Care and Use of Laboratory Animals from the National Academy of Sciences.

\section{Parasites and mammalian cells maintenance}

Leishmania (L.) infantum (MHOM/BR/1972/LD) promastigotes were maintained in M-199 medium supplemented with $10 \%$ fetal bovine serum (FBS) and $0.25 \%$ hemin at $24{ }^{\circ} \mathrm{C}$. The $L$. (L.) infantum amastigotes were maintained in golden hamsters for up to 60-70 days post-infection (d.p.i.) and isolated by differential centrifugation. NCTC (clone 929) murine conjunctive cells were maintained in RPMI-1640 (without phenol red) and supplemented with $10 \% \mathrm{FBS}$ at $37{ }^{\circ} \mathrm{C}$ in a humidified incubator containing $5 \% \mathrm{CO}_{2}$.
In vitro cytotoxic concentration $\left(\mathrm{CC}_{50}\right)$ against mammalian cells

The $50 \%$ cytotoxic concentration $\left(\mathrm{CC}_{50}\right)$ was determined in NCTC clone 929. NCTC cells were counted in a Neubauer hemocytometer and seeded at $6 \times 10^{4}$ cells per well in 96-well microplates at $37{ }^{\circ} \mathrm{C}$ in a $5 \% \mathrm{CO}_{2}$ incubator. AMD was then added in serial dilutions and tested for $48 \mathrm{~h}$; miltefosine was assayed as the standard drug. The cellular viability was determined by MTT assay at $570 \mathrm{~nm}$ [13]. For selectivity index (S.I.) the following equation was employed: S.I. $=\mathrm{CC}_{50} \mathrm{NCTC}$ cells $/ \mathrm{IC}_{50}$ amastigotes.

\section{In vitro inhibitory concentration $\left(\mathrm{IC} 5_{0}\right)$ against promastigotes and amastigotes}

To determine the $50 \%$ inhibitory concentration $\left(\mathrm{IC}_{50}\right)$ against $L$. (L.) infantum, promastigotes were counted in a Neubauer hemocytometer and seeded at $1 \times 10^{6}$ cells per well in 96-well microplates using miltefosine as the standard drug. AMD was added in serial dilutions and kept for $48 \mathrm{~h}$ at $24{ }^{\circ} \mathrm{C}$ until parasite viability had been determined by the MTT assay [13]. For $\mathrm{IC}_{50}$ determination against amastigotes, peritoneal macrophages were obtained by washing the peritoneal cavity of BALB/C mice with medium and seeded at $1 \times 10^{5}$ cells/well for $24 \mathrm{~h}$. Amastigotes were isolated from previously infected hamsters spleens, separated by differential centrifugation and added to the macrophages at a ratio of 1:10 (macrophage/amastigotes). Non-internalized parasites were removed by washing once with medium; and the cells were then incubated with AMD for $120 \mathrm{~h}$ at $37{ }^{\circ} \mathrm{C}$ in an incubator under $5 \% \mathrm{CO}_{2}$, using miltefosine as the standard drug. At the end of the assay, the cells were fixed in methanol, stained with Giemsa and observed under a light microscope to determine the number of intracellular parasites. The number of amastigotes was determined in 400 macrophages from the drug-treated and control wells [14].

\section{In vivo anti-Leishmania activity}

The efficacy of AMD treatment (oral and intraperitoneal) was determined using young male golden hamsters previously infected (i.p. route) with $L$. (L.) infantum amastigotes $\left(1 \times 10^{8} /\right.$ animal). Forty days post infection (d.p.i.), the hamsters ( $n=5$ /group) were treated intraperitoneally and orally for ten consecutive days with AMD at $50 \mathrm{mg} / \mathrm{kg} /$ day and at $100 \mathrm{mg} / \mathrm{kg} /$ day, respectively. The control group was treated with vehicle only. The animals were euthanized using $\mathrm{CO}_{2} 50$ d.p.i. and the parasite burden was evaluated by real time PCR using RNA samples obtained from the spleen and liver fragments, according to a standardized method published by Reimão and co-workers [15]. The susceptibility of Leishmania 
Table 1 In vitro IC 50 against promastigotes and amastigotes of Leishmania, CC $_{50}$ against NCTC cells and SI

\begin{tabular}{lllll}
\hline Drugs & Promastigotes & Amastigotes & Cytotoxicity \\
& $I C_{50}(\mu \mathrm{M})(95 \% \mathrm{Cl})$ & $I C_{50}(\mu \mathrm{M})(95 \% \mathrm{Cl})$ & $\mathrm{CC} 50(\mu \mathrm{M})(95 \% \mathrm{Cl})$ \\
\hline Amiodarone & 10.5 & 0.5 & 30.9 & $(12.5-76.1)$ \\
& $(9.5-11.7)$ & $(0.1-2.0)$ & $122.0(94.8-157.0)$ & 56.2 \\
Miltefosine & $16.8(15.4-17.5)$ & $17.8(11.6-24.6)$ & 12.8 \\
\hline
\end{tabular}

$I C_{50} 50 \%$ inhibitory concentration, $C_{50} 50 \%$ cytotoxicity concentration, $95 \%$ C.I. $95 \%$ confidence interval, S.I selectivity index

infantum to pentavalent antimony (Glucantime ${ }^{\circ}$ ) was previously determined in a hamster model [16].

\section{Statistical analysis}

All data obtained have been reported as the mean of two/ three independent assays. The $\mathrm{IC}_{50}$ values were calculated using sigmoid dose-response curves by the software Graph Pad Prism 5.0 (GraphPad Software Inc., La Jolla, $\mathrm{CA}$ ), and the 95\% confidence intervals were included in parentheses. ANOVA was used for statistical analysis.

\section{Results}

\section{In vitro anti-Leishmania activity $\left(\mathrm{IC}_{50}\right)$ and cytotoxicity} concentration $\left(\mathrm{CC}_{50}\right)$

The AMD assay performed against $L$. (L.) infantum promastigotes showed, after $48 \mathrm{~h}$ of incubation, an $\mathrm{IC}_{50}$ value of $10.5 \mu \mathrm{M}$. The drug was able to reduce by $100 \%$ the promastigotes viability at the highest concentrations. In order to determine the respective selectivity index for further intracellular investigation (amastigotes), the cytotoxicity assay was performed using NCTC cells, which presented a $\mathrm{CC}_{50}$ value of $30.9 \mu \mathrm{M}$ versus an $\mathrm{IC}_{50}$ value of $0.55 \mu \mathrm{M}$ for the amastigote form. AMD also exhibited a selectivity index of 56, relative to the intracellular form of the parasites. Miltefosine was used as the standard drug and the values are shown in Table 1.

\section{In vivo anti-Leishmania activity}

Considering the in vitro activity of AMD against intracellular amastigotes, the next step was to perform an in vivo experiment using the $L$. infantum-infected hamster model, in which after 40 d.p.i. the animals were treated for 10 consecutive days by two different routes of administration: intraperitoneal at $50 \mathrm{mg} / \mathrm{kg} /$ day and oral at $100 \mathrm{mg} / \mathrm{kg} /$ day. After 51 days of the initial infection, the animals were euthanized, spleen and liver fragments removed and the parasite burden was determined by qPCR.

The results demonstrated that AMD was not able to reduce the parasite burden in either the spleen or liver after intraperitoneal treatment. Although not significant $(p>0.05)$ a $114 \%$ increase in spleen infection and $38 \%$ in liver infection was observed in relation to the control group. In view of the oral treatment, AMD was also unable to reduce the infection in the spleen (5\% reduction); however, AMD was able to reduce the parasite burden by $20 \%$ in the liver, although without statistical significance $(p>0.05)$ (Fig. 1). The data below refer to a representative assay of two distinct experiments.

\section{Discussion}

Given the drug repositioning strategy and its great impact on leishmaniasis therapies - as demonstrated by the introduction of antimony, pentamidine, amphotericin B, azoles (ketoconazole, itraconazole, etc.), used initially to treat
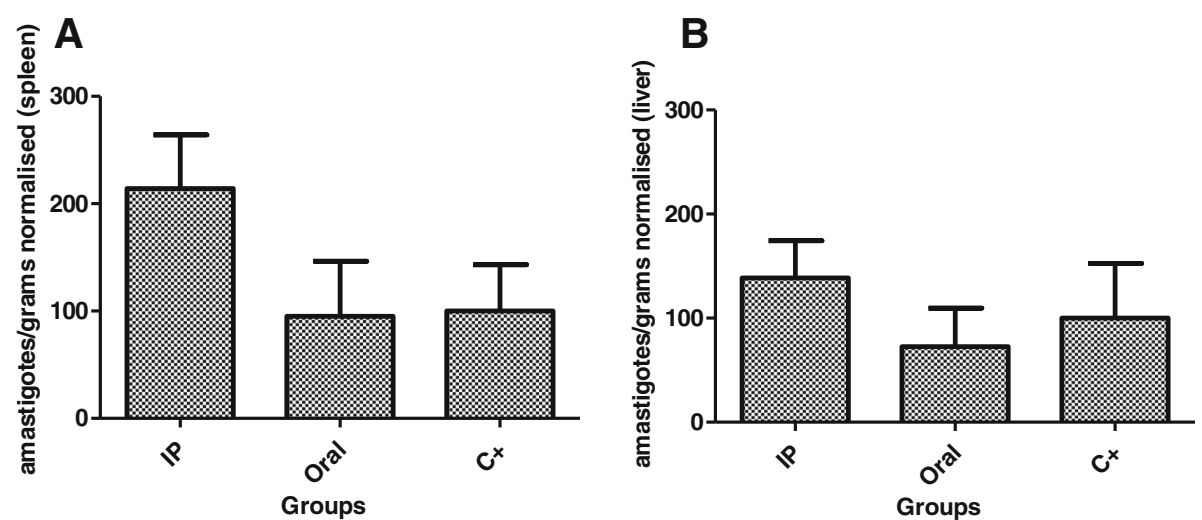

Fig. 1 In vivo evaluation of AMD in Leishmania-infected hamsters. The drug was administered for ten consecutive days at 50 mg/kg (i.p.) and $100 \mathrm{mg} / \mathrm{kg}$ (p.o.). Real-time PCR quantification (RNA amastigotes) of parasite burden in spleen (a) and liver (b). Data was normalized based in the vehicle group 
fungal infections [17], as well as miltefosine, developed for cancer treatment [18] - the drug amiodarone was chosen for the experimental trials due to its promising and unprecedented in vitro activity against $L$. (L.) infantum.

Considering the in vitro models, AMD showed a high selectivity index, demonstrating that the antiparasitic activity was approximately 56 times higher than the toxicity against mammalian cells. Additionally, AMD was about eight times more selective when compared to miltefosine, the standard oral treatment for VL in India. Based on the $\mathrm{IC}_{50}$ values against intracellular amastigotes, AMD was found to be approximately 32 times more potent than miltefosine. These findings corroborate the data described by Serrano-Martín et al. [10], in which they not only demonstrated promising selectivity of the drug AMD against L. mexicana, but also observed higher $\mathrm{IC}_{50}$ values in amastigotes $(8 \mathrm{nM})$ when compared to promastigotes (900 $\mathrm{nM}$ ).

The anti-Leishmania amazonensis [11], anti-Leishmania mexicana [10] and anti-Leishmania braziliensis [12] activities presented by AMD were previously described, both under in vitro (promastigotes and amastigotes) and in vivo models. Considering that different species may result in different sensitivities against the same drug [19], it was observed that AMD resulted in very similar $\mathrm{IC}_{50}$ values when compared to the species that cause tegumental or visceral diseases. This is exemplified by the finding by Nishikawa et al. [20] that AMD's anti- $L$. amazonensis activity resulted in an $\mathrm{IC}_{50}$ value of $0.46 \mu \mathrm{M}$, a fact that is extremely important when developing a drug not just for one disease but for a complex of diseases such as the complex of leishmaniases. However, the data presented in this paper to the best of our knowledge are unprecedented, since no study has been found in the literature demonstrating the potential of AMD against the fatal visceral form of leishmaniasis.

The anti-Trypanosoma cruzi activity of AMD has also been demonstrated through both in vitro and in vivo models, showing a mode of action related to mitochondrial damage and inhibition of ergosterol synthesis [21]. Amiodarone has also demonstrated an action that inhibits the calcium channels [22]; data from the literature have shown the activity of calcium channel blockers against amastigote forms of $L$. (L.) infantum [23]. Data reported by Paniz-Mondolfi et al. [12] demonstrated that AMD induced a "parasitological cure" in a single clinical case of $L .(V$.$) braziliensis when administered at the dose$ of $1,600 \mathrm{mg} /$ day for the first 4 days, followed by a dose of $800 \mathrm{mg} /$ day for 3 consecutive weeks. Additionally, an important fact to highlight associated with diseases caused by trypanosomatids and amiodarone use includes the drug prescription for chagasic patients that present cardiac compromise.
Taking into consideration the potential of this drug in the literature as well as its in vitro effectiveness, the study was carried out in an experimental hamster model of infection with $L$. (L.) infantum. Given the WHO criteria for new oral drug candidates for LV [5], the present study approached both oral and intraperitoneal administration. The results clearly demonstrated that after 10 days of treatment AMD was unable to induce any treatment in the target organs (spleen and liver), even when administered by two different routes (oral and intraperitoneal). Among the factors that could have contributed to the lack of effectiveness by the oral route, it is suggested that the absorption of the drug by the gastrointestinal tract is low and variable. Data from the literature report that only 20 to $55 \%$ of the drug is found in the circulation after oral administration [24]. In this case, future pharmaceutical formulations could be developed in an attempt to promote a better absorption of the drug, such as the production of AMD as nanocrystal, which favors an increase in the effectiveness of drugs, due to a better bioavailability [25]. Furthermore, the maximum oral dose used, namely $100 \mathrm{mg} / \mathrm{kg} /$ day, may not have been sufficient to achieve adequate plasma levels for parasite elimination. The literature describes the Lethal Dose $50 \%\left(\mathrm{LD}_{50}\right)$ after intravenous administration as $227 \mathrm{mg} / \mathrm{kg}$ in mice [26]; the use of higher doses might have caused toxicity in the animals, making the study inviable.

Another important factor that may have contributed to the lack of effectiveness in the animal model may be related to drug metabolism. AMD has been described as being extensively metabolized by the liver via CYP450, specifically by CYP2C8, resulting in a major metabolite, desethylamiodarone [27]. Although this metabolite continues to exhibit antiarrhythmic activity [28], nothing has been described in the literature as to its anti-Leishmania potential, a fact that should be investigated in an attempt to explain the absence of activity against the parasite in the experimental model.

AMD is an FDA-approved drug, is in conformity with Lipinsky's "Rule of Five" [29] and despite being in clinical use, presents problems related to its long half-life ( 58 days) [24] and consequent slow excretion may cause toxicity to the organism [30,31]. Although its pharmacokinetics profile is not ideal, the literature reports the daily use of AMD in patients, justifying in this work the dose regime choice in the experimental model. Considering the regimen adopted for both oral and intraperitoneal routes, it is possible to suggest that the drug may have contributed to animal toxicity, since a two-fold increase of parasite burden in spleen by intraperitoneal route was observed in relation to the control group. To corroborate this result, numerous studies in the literature describe AMD hepatotoxicity [32-34]. It is also 
important to highlight a case study that reported hepatic failure following AMD intraperitoneal administration at a dose of $750 \mathrm{mg}$ [32]. Additionally, the drug possesses extremely lipophilic properties with consequent accumulation in the liver, which results in tissue levels 500-fold higher in the liver than those found in the circulation [35]. Plomp and co-workers [36] demonstrated the high accumulation of the drug and metabolite in adipose tissues. Moreover, the toxicity expressed at higher doses following intraperitoneal administration of AMD may be associated with the action mode of the drug, which induces mitochondrial stress further aggravated by its metabolite desethylamiodarone [37].

In contrast, the literature provides reports of in vivo efficacy of AMD in a tegumental leishmaniasis model. Serrano-Martín et al. [38] demonstrated that after oral treatment with amiodarone at $50 \mathrm{mg} / \mathrm{kg} /$ day in an experimental model of L. mexicana, the drug produced superior efficacy compared to Glucantime. However, the authors reported that after drug decrease, reactivation of the disease was observed, indicating therapeutic failure [38]. Given the long half-life of AMD, combination therapy studies have been performed in VL therapy.

Serrano-Martín et al. [38] showed in vivo synergism of AMD when given in combination with miltefosine. The administration of amiodarone at $50 \mathrm{mg} / \mathrm{kg} / \mathrm{day}+$ miltefosine at $20 \mathrm{mg} / \mathrm{kg} /$ day resulted in a "parasitological cure" in $90 \%$ of the animals, as evaluated by optical microscopy, PCR and cell culture [38]. Furthermore Anversa et al. [39] showed that amiodarone used either on its own or in combination was unable to stop the development of cutaneous lesions caused by $L$. amazonensis; however, an improvement of pentavalent antimonial activity in the lesions has been observed with no side effects [39].

Additionally, data in the literature indicate that dronedarone may be a potential analog for future in vivo studies against $L$. ( $L$.) infantum because the compound is: i) a structural analogue of amiodarone; ii) has a lower half-life $(\sim 18 \mathrm{~h})$; iii) demonstrated in vitro values against L. mexicana more promising than amiodarone (promastigotes: $115 \mathrm{nM}$ versus $900 \mathrm{nM}$ /amastigotes: $0.65 \mathrm{nM}$ versus $8 \mathrm{nM}$ ) [40].

Finally, studies of drug delivery systems, such as phosphatidylserine-containing liposomes [41], may constitute a very promising alternative to target AMD at lower doses toward organs affected by the parasite, such as the liver, spleen and bone marrow.

\section{Conclusions}

Our study indicates that AMD is an in vitro potent FDA-approved drug against intracellular amastigotes of Leishmania (L.) infantum and may represent a lead compound for future synthesis of new analogues. Although the in vitro potency was clearly present in this compound, future analogues should also consider the reduction of the plasma half-life $\left(T_{1 / 2}\right)$, since long $T_{1 / 2}$ values can induce resistant parasites. However, these findings may serve as a basis for drug design studies directed at new AMD derivatives that could provide new candidates with an in vitro selectivity close to or even greater than that observed in the prototype seeking to deliver effectiveness in the experimental model of VL.

\begin{abstract}
Acknowledgments
We acknowledge Sao Paulo State Research Foundation (FAPESP) for support to E.G.P. (scholarship 2011/23703-1 and project 2015/23403-9) and Conselho Nacional de Pesquisa e Desenvolvimento (CNPq) for the scientific award to A.G.T (CNPq 306305/2017-8). We also thank Prof. Dr. Humberto G. Ferraz (University of Sao Paulo) for the amiodarone donation.
\end{abstract}

\section{Funding}

This publication was supported by the Coordination for the Improvement of Higher Education Personnel (CAPES) through Programa Editoração CAPES (edital n. 13/2016, auxílio n. 0722/2017, processo n.88881.142062/2017-01) and by the National Council for Scientific and Technological Development (CNPq) through Programa Editorial CNPq/CAPES 498 (chamada n. 26/2017, proc. n. 440954/2017-7)

\section{Availability of data and materials}

The datasets used and/or analysed during the current study are available from the authors on reasonable request.

\section{Authors' contributions}

EGP: conception, acquisition, analysis, interpretation of data and draft of manuscript. AGT: study conception and critical revision of manuscript. Both authors read and approved the final manuscript.

\section{Ethics approval}

All animal procedures were performed with the approval of the Research Ethics Commission (CEUA IAL 04/2011) and in agreement with the Guidelines for the Care and Use of Laboratory Animals from the National Academy of Sciences.

Consent for publication

Not applicable.

\section{Competing interests}

The authors declare that they have no competing interests.

\section{Publisher's Note}

Springer Nature remains neutral with regard to jurisdictional claims in published maps and institutional affiliations.

\section{Author details}

${ }^{1}$ Wellcome Centre for Anti-Infectives Research, School of Life Sciences, University of Dundee, Dundee, UK. ${ }^{2}$ Centre for Parasitology and Mycology, Instituto Adolfo Lutz, Avenida Dr. Arnaldo, 351, 8², Andar. Cerqueira César, São Paulo, SP CEP 01246-902, Brazil.

Received: 3 May 2018 Accepted: 5 October 2018

Published online: 25 October 2018

\section{References}

1. Feasey N, Wansbrough-Jones M, Mabey DC, Solomon AW. Neglected tropical diseases. Br Med Bull. 2010;93:179-200.

2. Nwaka S, Hudson A. Innovative lead discovery strategies for tropical diseases. Nat Rev Drug Discov. 2006;5(11):941-55.

3. Renslo AR, Mckerrow JH. Drug discovery and development for neglected parasitic diseases. Nat Chem Biol. 2006;2(12):701-10.

4. Sundar S, Chakravarty J. Investigational drugs for visceral leishmaniasis. Expert Opin Investig Drugs. 2015;24(1):43-59. 
5. World Health Organization. Control of the leishmaniases: report of a meeting of the WHO expert committee on the control of Leishmaniases. Geneva: WHO Technical report series; 2010.949.

6. Alvar J, Vélez ID, Bern C, Herrero M, Desjeux P, Cano J, et al. Leishmaniasis worldwide and global estimates of its incidence. PLoS One. 2012;7(5):e35671.

7. Johansen LM, DeWald LE, Shoemaker CJ, Hoffstrom BG, Lear-Rooney CM Stossel A, et al. A screen of approved drugs and molecular probes identifies therapeutics with anti-Ebola virus activity. Sci Transl Med. 2015;7(290):290ra89.

8. Nwaka S, Ridley RG. Virtual drug discovery and development for neglected diseases through public-private partnerships. Nat Rev Drug Discov. 2003; 2(11):919-28.

9. Singh BN. Amiodarone: historical development and pharmacologic profile. Am Heart J. 1983;106(4 Pt 2):788-97.

10. Serrano-Martín X, García-Marchan Y, Fernandez A, Rodriguez N, Rojas H, Visbal G, et al. Amiodarone destabilizes intracellular Ca2+ homeostasis and biosynthesis of sterols in Leishmania mexicana. Antimicrob Agents Chemother. 2009;53(4):1403-10.

11. de Macedo-Silva ST, de Oliveira Silva TLA, Urbina JA, de Souza W, Rodrigues JCF. Antiproliferative, ultrastructural, and physiological effects of amiodarone on promastigote and amastigote forms of Leishmania amazonensis. Mol Biol Int. 2011;2011:876021.

12. Paniz-Mondolfi AE, Pérez-Alvarez AM, Reyes-Jaimes O, Socorro G, Zerpa O, Slova D, et al. Concurrent Chagas' disease and borderline disseminated cutaneous leishmaniasis: the role of amiodarone as an antitrypanosomatidae drug. Ther Clin Risk Manag. 2008;4(3):659-63.

13. Tada H, Shiho O, Kuroshima K, Koyama M, Tsukamoto M. An improved colorimetric assay for interleukin 2. J Immunol Methods. 1986;93(2):157-65.

14. Yardley V, Croft SL. A comparison of the activities of three amphotericin B lipid formulations against experimental visceral and cutaneous leishmaniasis. Int J Antimicrob Agents. 2000;13(4):243-8.

15. Reimão JQ, Colombo FA, Pereira-Chioccola VL, Tempone AG. Effectiveness of liposomal buparvaquone in an experimental hamster model of Leishmania (L.) infantum chagasi. Exp Parasitol. 2012;130(3):195-9.

16. Tempone AG, Mortara RA, de Andrade HF Jr, Reimão JQ. Therapeutic evaluation of free and liposome-loaded furazolidone in experimental visceral leishmaniasis. Int J Antimicrob Agents. 2010;36(2):159-63.

17. Croft SL, Seifert K, Yardley V. Current scenario of drug development for leishmaniasis. Indian J Med Res. 2006;123(3):399-410.

18. Sundar S, Jha TK, Thakur CP, Engel J, Sindermann H, Fischer C, et al. Oral miltefosine for Indian visceral leishmaniasis. N Engl J Med. 2002;347(22):1739-46.

19. Croft SL, Sundar S, Fairlamb AH. Drug resistance in leishmaniasis. Clin Microbiol Rev. 2006;19(1):111-26.

20. Nishikawa K, Arai H, Inoue K. Scavenger receptor-mediated uptake and metabolism of lipid vesicles containing acidic phospholipids by mouse peritoneal macrophages. J Biol Chem. 1990;265(9):5226-31.

21. Benaim G, Sanders JM, Garcia-Marchán Y, Colina C, Lira R, Caldera AR, et al. Amiodarone has intrinsic anti-Trypanosoma cruzi activity and acts synergistically with posaconazole. J Med Chem. 2006;49(3):892-9.

22. Nishida A, Takizawa T, Matsumoto A, Miki T, Seino S, Nakaya H. Inhibition of ATP-sensitive $\mathrm{K}+$ channels and L-type Ca2+ channels by amiodarone elicits contradictory effect on insulin secretion in MIN6 cells. J Pharmacol Sci. 2011; 116(1):73-80.

23. Reimão JQ, Scotti MT, Tempone AG. Anti-leishmanial and anti-trypanosomal activities of 1,4-dihydropyridines: in vitro evaluation and structure-activity relationship study. Bioorg Med Chem. 2010;18(22):8044-53.

24. DrugBank. Amiodarone. Available from: http://www.drugbank.ca/drugs/DB01118.

25. Jinno J, Kamada N, Miyake M, Yamada K, Mukai T, Odomi M, et al. Effect of particle size reduction on dissolution and oral absorption of a poorly watersoluble drug, cilostazol, in beagle dogs. J Control Release. 2006;111(1-2):56-64.

26. Barle EL, Cerne M, Peternel L, Homar M. Reduced intravenous toxicity of amiodarone nanosuspension in mice and rats. Drug Chem Toxicol. 2013; 36(3):263-9

27. Ohyama K, Nakajima M, Nakamura S, Shimada N, Yamazaki H, Yokoi T. A significant role of human cytochrome P450 2C8 in amiodarone Ndeethylation: an approach to predict the contribution with relative activity factor. Drug Metab Dispos. 2000;28(11):1303-10.

28. Morvay N, Baczkó I, Sztojkov-Ivanov A, Falkay G, Papp JG, Varró A, et al. Long-term pretreatment with desethylamiodarone (DEA) or amiodarone (AMIO) protects against coronary artery occlusion induced ventricular arrhythmias in conscious rats. Can J Physiol Pharmacol. 2015;93(9):773-7.
29. Zhang MQ, Wilkinson B. Drug discovery beyond the 'rule-of-five'. Curr Opin Biotechnol. 2007;18(6):478-88.

30. Pomponio G, Savary CC, Parmentier C, Bois F, Guillouzo A, Romanelli L, et al. In vitro kinetics of amiodarone and its major metabolite in two human liver cell models after acute and repeated treatments. Toxicol in Vitro. 2015;30(1 Pt A):36-51.

31. Buggey J, Kappus M, Lagoo AS, Brady CW. Amiodarone-induced liver injury and cirrhosis. ACG Case Rep J. 2015:2(2):116-8.

32. Cataldi A, Gonella D, Robutti N, Siri M, Buonocore S, Odetti P. Hepatotoxicity after intravenous amiodarone. Aging Clin Exp Res. 2008;20(6):593-6.

33. Cimic A, Sirintrapun J. Amiodarone hepatotoxicity with absent phospholipidosis and steatosis: a case report and review of amiodarone toxicity in various organs. Case Rep Pathol. 2013;2013:201095.

34. Turk U, Turk BG, Yılmaz SG, Tuncer E, Alioğlu E, Dereli T. Amiodaroneinduced multiorgan toxicity with ocular findings on confocal microscopy. Middle East Afr J Ophthalmol. 2015;22(2):258-60.

35. Gassanov N, Caglayan E, Erdmann E, Er F. Amiodarone-induced liver dysfunctions. Dtsch Med Wochenschr. 2010;135(27):1372-4.

36. Plomp TA, Wiersinga WM, Van Rossum JM, Maes RA. Pharmacokinetics and body distribution of amiodarone and desethylamiodarone in rats after oral administration. In Vivo. 1987;1(5):265-79

37. Takai S, Oda S, Tsuneyama K, Fukami T, Nakajima M, Yokoi T. Establishment of a mouse model for amiodarone-induced liver injury and analyses of its hepatotoxic mechanism. J Appl Toxicol. 2016;36(1):35-47.

38. Serrano-Martín X, Payares G, De Lucca M, Martinez JC, Mendoza-León A, Benaim G. Amiodarone and miltefosine act synergistically against Leishmania mexicana and can induce parasitological cure in a murine model of cutaneous leishmaniasis. Antimicrob Agents Chemother. 2009; 53(12):5108-13

39. Anversa L, Salles Tiburcio MG, Batista LR, Cuba MB, Nogueira Nascentes GA, Martins TY, et al. Amiodarone and itraconazole improve the activity of pentavalent antimonial in the treatment of experimental cutaneous leishmaniasis. Int J Antimicrob Agents. 2017;50(2):159-65.

40. Benaim G, Casanova P, Hernandez-Rodriguez V, Mujica-Gonzalez S, ParraGimenez N, Plaza-Rojas L, et al. Dronedarone, an amiodarone analog with improved anti-Leishmania mexicana efficacy. Antimicrob Agents Chemother. 2014:58(4):2295-303.

41. Pinto EG, da Costa-Silva TA, Tempone AG. Histamine H1-receptor antagonists against Leishmania (L.) infantum: an in vitro and in vivo evaluation using phosphatidylserine-liposomes. Acta Trop. 2014;137:206-10.

Ready to submit your research? Choose BMC and benefit from:

- fast, convenient online submission

- thorough peer review by experienced researchers in your field

- rapid publication on acceptance

- support for research data, including large and complex data types

- gold Open Access which fosters wider collaboration and increased citations

- maximum visibility for your research: over $100 \mathrm{M}$ website views per year

At $\mathrm{BMC}$, research is always in progress.

Learn more biomedcentral.com/submission 